\title{
Protostellar turbulence in cluster forming regions of molecular clouds
}

\author{
Fumitaka Nakamura ${ }^{1}$ and Zhi-Yun $\mathrm{Li}^{2}$ \\ ${ }^{1}$ Faculty of Education and Human Sciences, Niigata University, 8050 Ikarashi-2, Niigata \\ 950-2181, Japan, email: fnakamur@ed.niigata-u.ac.jp \\ ${ }^{2}$ Department of Astronomy, University of Virginia, P.O. Box 400325, Charlottesville, VA \\ 22904, USA, email: zl4h@virginia.edu
}

\begin{abstract}
We perform 3D MHD simulations of cluster formation in turbulent magnetized dense molecular clumps, taking into account the effect of protostellar outflows. Our simulation shows that initial interstellar turbulence decays quickly as several authors already pointed out. When stars form, protostellar outflows generate and maintain supersonic turbulence that have a powerlaw energy spectrum of $E_{k} \sim k^{-2}$, which is somewhat steeper than those of driven MHD turbulence simulations. Protostellar outflows suppress global star formation, although they can sometimes trigger local star formation by dynamical compression of pre-existing cores. Magnetic field retards star formation by slowing down overall contraction. Interplay of protostellar outflows and magnetic field generates large-amplitude Alfven and MHD waves that transform outflow motions into turbulent motions efficiently. Cluster forming clumps tend to be in dynamical equilibrium mainly due to dynamical support by protostellar outflow-driven turbulence (hereafter, protostellar turbulence).
\end{abstract}

Keywords. ISM: magnetic fields, ISM: clouds, MHD, stars: formation, turbulence

\section{Introduction}

The majority of stars are thought to form in clusters. Observations show that cluster forming regions are strongly influenced by supersonic turbulence. Also, molecular clouds are known to be magnetized. Observed magnetic energy is comparable to gravitational and turbulent kinetic energies, suggesting that the magnetic field as well as supersonic turbulence is dynamically important in cloud evolution. On the other hand, numerical simulations have demonstrated that supersonic turbulence decays quickly, on a timescale comparable to the turbulence crossing time on the dominant energy-carrying scale, with or without a strong magnetic field (e.g., Stone et al. 1998; MacLow et al. 1998). Therefore, the supersonic turbulence must be replenished somehow. One promising mechanism of turbulence supply in cluster-forming clumps is protostellar outflows, which are observed in abundance in nearby cluster-forming regions. In fact, there is strong evidence that protostellar outflows strongly affect cloud dynamics and star formation in several regions (e.g., NGC1333: Quillen et al. 2005; Circinus cloud: Bally et al. 1999). However, it is not well-understood how protostellar outflows affect or control cloud dynamics and star formation in cluster-forming clumps. We have made a start in numerical study of cluster formation including spherical protostellar outflows (Li \& Nakamura 2006). Here we present the results of new simulations that take into account the outflow collimation. See Nakamura \& Li (2006) for more detail.

\section{Numerical model}

We consider a centrally condensed self-gravitating spherical isothermal cloud in a cubic box of length $L=9 L_{J}$, where $L_{J}$ is the thermal Jeans length. The radius of the central region of nearly constant density is set to $1.5 L_{J}$. The initial cloud is threaded by a 
uniform magnetic field whose strength $\left(B_{0}\right)$ is specified by $\alpha \equiv B_{0}^{2} /\left(8 \pi c_{s}^{2} \rho_{0}\right)$, where $\rho_{0}$ is the central density and $c_{s}$ is the isothermal sound speed. For the standard model presented below, we set $\alpha=2.5$. The cloud is magnetically supercritical: the averaged magnetic flux-to-mass ratio is about a half the critical value. At the beginning of the simulation, we impose on the cloud a supersonic velocity field of power spectrum $E_{k} \propto$ $k^{-1}$. The initial rms turbulent Mach number is set to $\mathcal{M}=10$. We follow the cloud evolution with a 3D MHD code based on Roe's TVD method.

Initial turbulence generates small self-gravitating cores, from which several stars form. In our simulations, star formation is treated as follows. When the central density of a dense self-gravitating core exceeds a threshold density $\left(\rho_{\text {th }}=100 \rho_{0}\right)$, a fraction $\epsilon(=20 \%)$ of its mass is converted into a Lagrangian point particle that represents a newborn star. At the same time, the outflow momentum is added in the surrounding gas. The protostellar outflow is assumed to be bipolar, and its direction is parallel to the local magnetic field line. The half opening angle of the protostellar jet component is chosen to be 30 degree. The outflow strength is determined by two parameters: the stellar mass $M_{*}$ and a dimensionless factor $f$ (Nakamura \& Li 2005). The stellar mass is automatically determined when the core mass is identified, $M_{*}=\epsilon M_{\text {core }}$. The factor $f$ is uncertain, and may lie in the range $\sim 0.1-1.0$ (see Nakamura \& Li 2006 for more detail). We adopt for the standard model presented below $f=0.5$, corresponding to an outflow momentum of $50 M_{\odot} \mathrm{km} / \mathrm{s}$ per solar mass of stellar material.

\section{Numerical results}

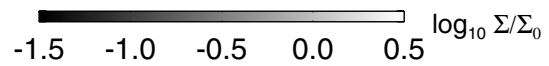

(a): $\mathrm{t}=0.5 \mathrm{t}_{\mathrm{g}}$

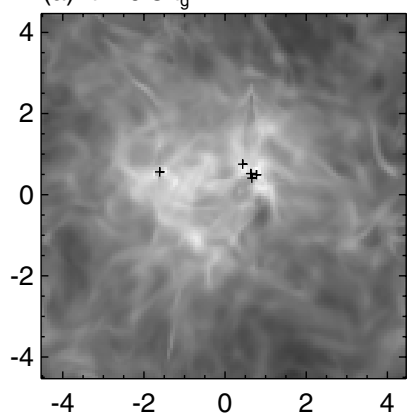

(b): $t=1.0 t_{g}$

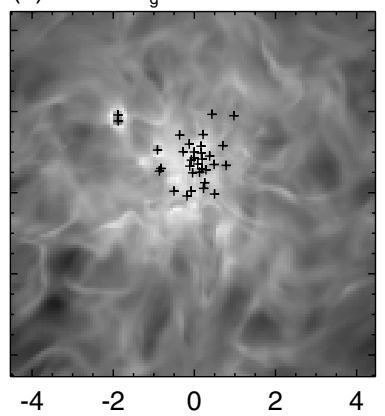

(c): $t=1.5 t_{g}$

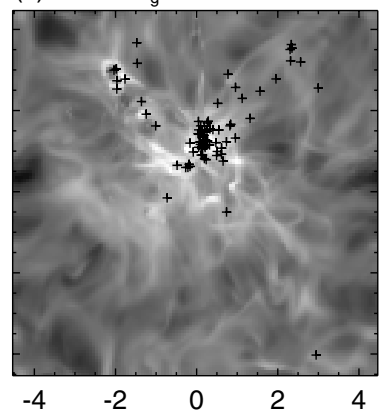

Figure 1. Column density distributions for the model with $\alpha=2.5, \mathcal{M}=10$, and $f=0.5$ at three different times: (a) $t=0.5 t_{g}$, (b) $1.0 t_{g}$, and (c) $1.5 t_{g}$. Crosses indicate the positions of stars.

As a typical example, we show in Figure 1 the evolution of the model with $\alpha=2.5$, $\mathcal{M}=10$, and $f=0.5$. At early times, initial turbulence decays quickly as several authors demonstrated. The first star forms at $t \sim 0.4 t_{g}$, where $t_{g}$ is the gravitational collapse time defined as $L_{J} / C_{s}=\left(\pi / G \rho_{0}\right)^{1 / 2}$ (roughly $25 \%$ longer than the global free fall time). After that, several stars form from the dense fragments generated by the initial turbulent compression [panel (a)]. However, most stars form in high-density regions disturbed strongly by outflows [panels (b) and (c)]. At late times, the velocity dispersion reaches about $\left\langle\Delta V^{2}\right\rangle^{1 / 2} \simeq 5 c_{s} \simeq 1.5(T / 20 \mathrm{~K})^{1 / 2} \mathrm{~km} \mathrm{~s}^{-1}$. This is consistent with the observed values in nearby cluster forming regions such as NGC1333. Our simulation indicates that protostellar outflows influence cloud dynamics and star formation significantly. We note that several stars form by dynamical compression due to outflows. In other words, local star formation can be triggered by outflows. 
To assess the effect of protostellar outflows on star formation, we depict in the left panel of Figure 2 the time evolution of star formation efficiency (SFE) for the $\alpha=2.5$ models with three different outflow strengths: $f=0.25,0.5$, and 0.75 . Here, the SFE is defined as the ratio of the total stellar mass divided by the total mass of stars and gas. For the model presented in Figure 1, the SFE reaches $6 \%$ by $2 t_{g}$. This corresponds to the star formation rate per unit free-fall time of $\mathrm{SFR}_{\mathrm{ff}} \simeq 3 \%$ (see Krumholz \& Tan $2006)$. For the model with weaker outflows $(f=0.25)$, the SFE reaches about $10.5 \%$ by $2 t_{g}\left(\mathrm{SFR}_{\mathrm{ff}} \simeq 5.3 \%\right)$. For the stronger outflow case $(f=0.75)$, the SFE reaches $3 \%$ by $1.9 t_{g}\left(\mathrm{SFR}_{\mathrm{ff}} \simeq 1.5 \%\right)$. Thus, we conclude that the outflows tend to suppress global star formation. We note that this agrees with the results of driven-turbulence simulations where driven turbulence tends to reduce star formation efficiency (e.g., Vazquez-Semadeni et al. 2005). To see how SFE depends on initial magnetic field strength, we show in the right panel of Figure 2 three models with $\alpha=2.5,0.5$ and $10^{-6}$. Clearly, magnetic field also suppresses the global star formation. We conclude that both protostellar outflow and magnetic field are important in regulating the rate of star formation in cluster forming regions.
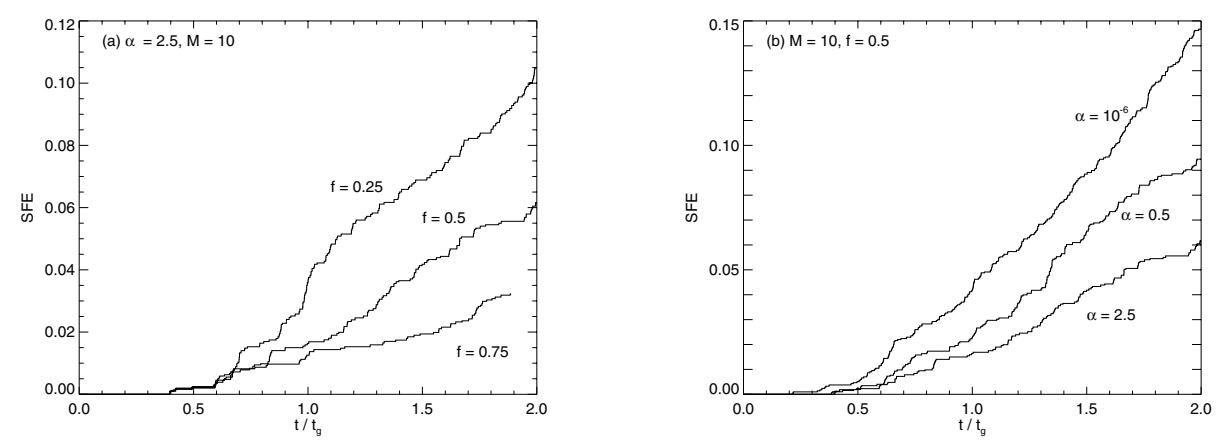

Figure 2. Time evolution of star formation efficiency (SFE). left: SFEs for three models with different outflow strength: $f=0.25,0.5$, and 0.75 . Protostellar outflows tend to suppress global star formation. right: SFEs for three models with different magnetic field strength: $\alpha=10^{-6}, 0.5$, and 2.5. The outflow strength is set to $f=0.5$. The magnetic field also retards star formation significantly.

To see the dynamical state of the cloud, we define a net radial infall speed from $v_{\text {net }}=\dot{M}_{\text {net }}(r) / 4 \pi r^{2} \tilde{\rho}(r)$, where $\dot{M}_{\text {net }}(r)$ is the net mass flux through a sphere of radius $r$ centered on the minimum of gravitational potential, and $\tilde{\rho}(r)$ is the average mass density at that radius. In the left panel of Figure 3, we plot $v_{\text {net }}$ as a function of radius at several representative times: $0.5,1.0,1.5$, and $2.0 t_{g}$. Note that the net mass flow can either be infall or outflow at a given radius. Its magnitude is, however, generally comparable to, or smaller than the isothermal sound speed. For comparison, the characteristic gravitational speed is $\left.v_{g}=[G M /(L / 2))\right]^{1 / 2}=8.72 c_{s}$, which is much larger than the net infall or outflow speed. Therefore, the average mass redistributes at a speed much less than the characteristic dynamical speed, which is a clear indication that the system has reached a dynamical equilibrium. The radial density profile is approximated by a power-law of $\rho \propto r^{-1.5}$ during the evolution (the right panel of Figure 3).

Our simulations also indicate that protostellar outflows generate large-amplitude MHD waves by strongly distorting magnetic field lines. The waves seem to be important in transforming the outflow motions into local turbulent motions. The kinetic energy power spectrum shows a power-law of $E_{k} \propto k^{-n}$, with $n \approx 2$. The value of $n$ is more or less independent of the power index of the initial turbulent field. The power index of protostellar 

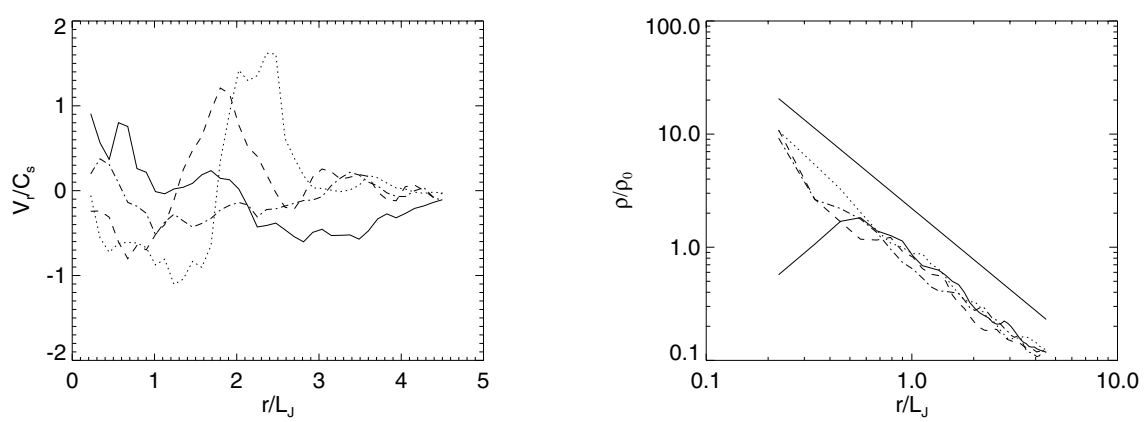

Figure 3. left: Net radial velocity normalized by isothermal sound speed. It is measured from the distance from the cluster center. The cluster center is determined as the point where the gravitational potential takes its minimum. right: Radial density profile normalized by $\rho_{0}$. Straight line denotes the power-law profile of $r^{-1.5}$.

turbulence seems to be somewhat steeper than those of driven MHD turbulence simulations such as Boldyrev et al. (2002)'s $(n=1.74)$.

\section{Summary}

We have carried out 3D numerical simulations of cluster formation taking into account supersonic turbulence, magnetic fields, and protostellar outflows. We found that although initial interstellar turbulence decays very quickly, protostellar outflows can drive and maintain supersonic turbulence in cluster forming clumps. In the model presented above, the velocity dispersion stays around $\left\langle\Delta V^{2}\right\rangle^{1 / 2} \sim 5 c_{s} \simeq 1.5(T / 20 \mathrm{~K})^{1 / 2} \mathrm{~km} \mathrm{~s}^{-1}$, which is in good agreement with the observed values in nearby cluster forming regions. Stars form in high-density regions disturbed strongly by protostellar outflows. We call this outflow-driven turbulence "protostellar turbulence". Protostellar outflows suppress global star formation, although they can sometimes trigger local star formation, e.g., due to dynamical compression of pre-existing dense cores. Our simulations indicate that clusterforming clumps are close to a dynamical equilibrium. Star formation rate per unit freefall time $\left(\mathrm{SFR}_{\mathrm{ff}}\right)$ stays around a few percent, which is in good agreement with recent estimates of this number from a range of observations (Krumholz \& Tan 2006).

\section{Acknowledgements}

Numerical calculations are carried out on NEC SX6 at Niigata University, Hitachi SR8000 at the Center for Computational Science, University of Tsukuba and NEC SX8 at Yukawa Institute, Kyoto University. The research is supported in part by NSF AST0307368, NASA NNG05GJ49G, and the Grant-in-Aid (18540234) from MEXT of Japan.

\section{References}

Bally, et al. 1999, AJ 117, 410

Boldyrev, S., Nordlund, A. \& Padoan, P. 2002, ApJ 573, 678

Li, Z.-Y. \& Nakamura, F. 2006, ApJ 640, L187

Krumholz M. \& Tan, J. 2006, astro-ph/0606277

MacLow, M.-M., Klessen, R., Burkert, A. \& Smith, M.D. 1998, PRL 80, 2754

Nakamura, F. \& Li, Z.-Y. 2005, ApJ 631, 411

Nakamura, F. \& Li, Z.-Y. 2006, in preparation

Quillen, et al. 2005, ApJ 632, 941

Stone, J.M., Ostriker, E.C. \& Gammie, C.F. 2005, ApJ 508, L99

Vazquez-Semadeni, E., et al. 2005, ApJ 630, 49 


\section{Discussion}

Hily-Blant: Since outflows drive turbulence according to your MHD simulations, and since the star formation efficiency decreases with outflow feedback, how do your models compare to Padoan and Vazquez-Semadeni simulations in terms of power spectra?

NAKAMURA: We are now analyzing the power spectrum of our model. Preliminary results show that the energy power spectrum of outflow models follows $E_{k} \propto k^{-2}$, whereas the power spectrum of no outflow models is steeper than $E_{k} \propto k^{-2}$. Therefore, the slope of the power spectrum of outflow models is more or less consistent with Padoan's. However, we haven't done any quantitative comparison with Padoan's model yet. As for Enrique's work, our simulation is in good agreement with his results because turbulence driven by outflows tends to suppress star formation.

E. Ostriker: (1) Have you quantified the difference in efficiency for transferring outflow energy to turbulence in magnetized vs. unmagnetized models? (2) Have you measured the effective pressure as a function of radius, i.e., $\left\langle\rho v^{2}\right\rangle(r)$ and $\left\langle B^{2}\right\rangle(r)$ ?

NAKAmura: (1) No, (2) Not yet. I showed that the cloud is in dynamical equilibrium and the thermal pressure is much smaller than turbulent pressure. Therefore, turbulent pressure and magnetic force should contribute to force balance. 